\title{
Addition of probiotics to glutamine therapy as intestinal barrier protection for severe surgical patients.
}

\author{
Baohua Wang*, Yinhua Wang, Yingang Zhang, Haixia Chai, Xingwei Xuan \\ The Affiliated Hospital of North China University of Science and Technology, Tangshan City, PR China
}

\begin{abstract}
Objective: To study the protecting effect of combining probiotics with Glutamine (GIn) in intestinal barrier function of severe surgical patients

Methods: A total of 36 surgical patients with severe gastrointestinal diseases were randomly divided into routine Parenteral Nutrition (PN) group, probiotics group, and combination group, which received conventional PN, PN+probiotics and PN+probiotics+GLn respectively. Then all groups were measured the ratio of lactulose and Mannitol (L/M), the concentration of serum endotoxin, the level of intestinal Lactobacillus, and the number of gram-negative bacterium before operation and at the $3^{\text {rd }}, 8^{\text {th }}$ day after operation

Results: At $8^{\text {th }}$ day, the ratio of lactulose and mannitol levels as well as the concentration of serum endotoxin in the probiotics group were much lower than those in the $P N$ group $(P<0.05)$, but much higher than the combination group $(\mathrm{P}<0.05)$ The level of intestinal Lactobacillus in the probiotics group was much higher than that in the $P N$ group $(P<0.05)$, but lower than the combination group $(P<0.05)$. There was no significant difference in the number of gram-negative bacterium among the three groups (P>0.05).

Conclusions: Addition of probiotics to Gln therapy has better protecting effect in intestinal barrier function.
\end{abstract}

Keywords: Probiotics, Glutamine, Intestinal barrier function.

Accepted on May 11, 2017

\section{Introduction}

In general, the patients with severe surgical disease have malnutrition, especially those elderly patients with weak constitution, which damages the intestinal barrier function easily, even leads to Multiple Organ Dysfunction Syndromes (MODS) [1]. It is confirmed already that glutamine have protecting effect for intestinal barrier function. What's more the people have been paying attention to the protection of probiotics for gut barrier. However, there are few researches about the combination of them in protecting the gut barrier of the patients with severe surgical diseases, reducing the intestinal permeability, improving the diversity of gastrointestinal flora, and decreasing endotoxin [2,3]. This study is to explore the protecting effect of combining probiotics with Gln in intestinal barrier function of severe surgical patients.

\section{Materials and Methods}

\section{Subjects and groups}

A total of 36 cases with severe diseases admitted in our department from December 2010 to May 2011 were enrolled, 18 female, 18 male. All of them had no problems of heart, liver, kidney, and lung, as well as taboo of enteral nutrition. The participants were randomly divided into routine Parenteral Nutrition (PN) group, probiotics group, and combination group, 6 male and 6 female a group. In the PN group, all patients, who were between 30 and 70 years old, $54.5 \pm 7.2$ in average, and $15.4 \pm 1.8$ in the scores of APACHEII, received routine parenteral nutrition. In the probiotics group, all patients, who were between 28 and 68 y old, $53.4 \pm 6.8$ in average, and $15.6 \pm 1.7$ in the scores of APACHEII, accepted PN and probiotics. In the combination group, all patients, who were between 29 and $69 \mathrm{y}$ old, $53.8 \pm 6.4$ in average, and 15.6 \pm 1.7 in the scores of APACHEII, received PN and probiotics and GLn. There were no significant difference in sex, age, and scores of APACHEII in three groups $(\mathrm{P}>0.05)$. Ethics approval of the study was obtained from the local research ethics committee, and all participants signed an informed consent voluntarily.

\section{Treatment methods}

After correcting the abnormal hemodynamics, acid-base imbalance, electrolyte disorder and pathoglycemia, with stable life signs, all participants were given nutritional support and probiotics immediately. Three groups were given routine parenteral nutrition, and the probiotics group also took Live 
Combined Bifidobacterium and Lactobacillus tablets (brand name: Golden Bifid, Inner Mongolia Shuang Qi pharmaceutical Co., Ltd.), 4 tablets a time, three times a day, the combination group as well. In addition, the combination group received alanyl-glutamine (Hainan Tongyong Kangli pharmaceutical Limited) intravenously, $20 \mathrm{~g}$ Qd. The whole treatment course was $8 \mathrm{~d}$.

\section{Observation indexes}

Venous blood was obtained before operation on $\mathrm{d} 3$ and 8 after operation. The endotoxin was measured with chromogenic technique of limulus test and spectrophotometer, and the value of L/M was determined using high pressure liquid chromatography of electrochemical detector. Fresh specimens of stool were put into the sterilized sodium chloride solution and then were diluted. These mixed solutions were dropped to the selective medium to analyse qualitatively and quantitatively. Sugar fermentation medium was used for probiotics, and blood agar culture-medium for gram-negative bacterium.

\section{Statistical analysis}

The data was processed using statistical analysis software SAS 9.2. The data was expressed with $\mathrm{x} \pm \mathrm{S}$, and analysed by analysis of variance (ANOVA) method and $t$ test. The statistical significance was defined as $\mathrm{P}<0.05$.

\section{Results}

\section{General condition after nutritional therapy}

There was one case with incision infection in combination group, and one case with diarrhea in the probiotics group. Apart from these, there was no case with abdominal pain, diarrhea, and incision infection, etc.

\section{Intestinal permeability}

The comparison of L/M in three groups before and after operation was seen in Table 1. Before operation and on three day after operation, compared L/M of urination in three groups, there was no statistic difference $(\mathrm{P}>0.05)$. On eight day, with postoperative nutrition support, $\mathrm{L} / \mathrm{M}$ in probiotics group was much lower than that in $\mathrm{PN}$ group $(\mathrm{P}=0.0043)$, but higher than the combination group $(\mathrm{P}=0.041)$, indicating that the intestinal permeability was improved.

Table 1. Comparison of the $L / M$ in three groups before and after operation.

\begin{tabular}{|c|c|c|c|}
\hline Group & Before operation & $\begin{array}{l}\text { On d } 3 \text { after } \\
\text { operation }\end{array}$ & $\begin{array}{l}\text { On d } 8 \text { after } \\
\text { operation }\end{array}$ \\
\hline PN group & $0.122 \pm 0.013$ & $0.134 \pm 0.024$ & $0.146 \pm 0.015$ \\
\hline Probiotics group & $0.125 \pm 0.016$ & $0.116 \pm 0.031$ & $0.092 \pm 0.043^{a}$ \\
\hline Combination & $0.123 \pm 0.015$ & $0.103 \pm 0.027$ & $0.078 \pm 0.025^{\mathrm{b}}$ \\
\hline
\end{tabular}

Notes: a was compared with $\mathrm{PN}$ group, $\mathrm{p}=0.043$; $\mathrm{b}$ was compared with probiotics group, $p=0.0041$

\section{Serum endotoxin}

The comparison of serum endotoxin in three groups before and after operation was seen in Table 2. Before operation and on $\mathrm{d}$ 3 after operation, compared the serum endotoxin in three groups, there was no statistic difference $(\mathrm{P}>0.05)$. On $\mathrm{d} 8$, with postoperative nutritional therapy, $\mathrm{L} / \mathrm{M}$ in probiotics group was much lower than that in $\mathrm{PN}$ group $(\mathrm{P}=0.037)$, but higher than the combination group $(\mathrm{P}=0.043)$.

Table 2. Comparison of the level of serum endotoxin $(n g / L)$ in three groups before and after operation.

\begin{tabular}{llll}
\hline Group & Before operation & $\begin{array}{l}\text { On } \mathbf{d} 3 \text { after } \\
\text { operation }\end{array}$ & $\begin{array}{l}\text { On } \mathbf{d} \text { after } \\
\text { operation }\end{array}$ \\
\hline PN group & $0.382 \pm 0.016$ & $0.523 \pm 0.027$ & $0.446 \pm 0.035$ \\
\hline Probiotics group & $0.376 \pm 0.031$ & $0.476 \pm 0.012$ & $0.312 \pm 0.023^{\mathrm{a}}$ \\
\hline $\begin{array}{l}\text { Combination } \\
\text { group }\end{array}$ & $0.387 \pm 0.024$ & $0.421 \pm 0.019$ & $0.298 \pm 0.032^{\mathrm{b}}$ \\
\hline
\end{tabular}

Notes: a was compared with $\mathrm{PN}$ group, $\mathrm{p}=0.037$; $\mathrm{b}$ was compared with probiotics group, $p=0.043$

Table 3. Logarithm comparison of the intestinal Lactobacillus and the gram-negative bacterium concentration in patients' defection of three groups before and after operation.

\begin{tabular}{llll}
\hline Bacteria genus & Time & PN group & Probiotics group \\
\hline Lactobacillus & Before operation & $7.43 \pm 0.16$ & $7.36 \pm 0.27$ \\
\cline { 2 - 4 } & On d 3 after operation & $7.46 \pm 0.13$ & $7.53 \pm 0.21$ \\
\cline { 2 - 4 } & On d 8 after operation & $7.52 \pm 0.11$ & $8.36 \pm 0.18^{\mathrm{a}}$ \\
\hline Gram-negative bacterium & Before operation & $8.23 \pm 0.21$ & $8.28 \pm 0.25$ \\
\cline { 2 - 4 } & On d 3 after operation & $8.42 \pm 0.32$ & $8.35 \pm 0.17$ \\
\cline { 2 - 4 } & On d 8 after operation & $8.35 \pm 0.43$ & $8.30 \pm 0.21$ \\
\hline
\end{tabular}

Notes: a was compared with PN group, $p=0.038$; $b$ was compared with probiotics group, $p=0.035$; $c$ was compared with $P N$ group, $p=0.082$; $d$ was compared with probiotics group, $\mathrm{p}=0.068$. 


\section{Change of intestinal flora in patients' defecation before and after operation}

The logarithm comparison of the intestinal Lactobacillus and the gram-negative bacterium concentration in patients' defection of three groups before and after operation was seen in Table 3. Before operation and on three day after operation, compared the intestinal flora in three groups, there was no statistic difference $(\mathrm{P}>0.05)$. On d 8 after operation, the concentration of intestinal Lactobacillus in the probiotics group and combination group were much higher than that in $\mathrm{PN}$ group $(\mathrm{P}=0.038)$, and the combination was also much higher than the probiotics group $(\mathrm{P}=0.0035)$. However, there was no significant difference in the level of gram-negative bacterium of three groups $(\mathrm{P}>0.05)$. The colony count was expressed by the value of logarithm $(\operatorname{lgCFU} / \mathrm{g})$.

\section{Discussion and Conclusions}

This study proved that with simple parenteral nutritional therapy, before operation and on the $\mathrm{d} 3$ and 8 after operation, the intestinal permeability still increased, the serum endotoxin also rose, unprotecting the intestinal barrier function effectively. Combining with probiotics, there was no difference before operation and on $\mathrm{d} 3$ after operation. But on $\mathrm{d} 8$, the $\mathrm{L} / \mathrm{M}$ and serum endotoxin in probiotics group were much lower than those in PN group, indicating that probiotics help to improve the intestinal permeability and decrease endotoxin translocation. That may be related to that using probiotics increased the number of Lactobacillus, alleviated the damage of intestinal ecological protective screen, mechanical barrier restored, and inhibited the growth of the gram-negative bacterium inhibited. Through measured colony count of flora in patients' defecation, it was confirmed.

Due to addition of Gln and probiotics, which had synergistic effect, provided energy to intestinal mucous cells, but also improved the diversity of intestinal flora, before operation and on $\mathrm{d} 3$ and 8 after operation. L/M and the level of serum endotoxin in the combination group were much lower than the other two groups, and the concentration of Lactobacillus was much higher. Thereby, they protected the intestinal barrier function. Before operation and on $\mathrm{d} 3$ and 8 after operation, the level of gram-negative bacterium in all groups had no difference, indicating that probiotics could not kill harmful germs or potential pathogenic bacteria directly, however, the increasing and using of probiotics improved the structure of intestinal flora, reduced the proportion of gram-negative bacterium, and benefited the health of intestine and the body.

Intestine is the biggest and most complex organ in human body, and the intestinal mucosal barrier refers to the largest one which prevents the translocation of bacteria and endotoxin in enteric cavity [4]. Normally, there are four intestinal mucosal barriers [5], including mechanical barrier, ecological barrier, chemical barrier, and immune barrier, which form a complex but ordered barrier net. The lack of any link in this net may affect the normal protecting effect of intestinal barrier. The gastrointestinal barrier can promote blood and oxygen supply to intestine, and provide nutritional support, which are its major two protecting methods [6]. This study uses the addition probiotics to Gln therapy to protect the function of intestinal barrier.

When preparing the bowel for the surgery $3 \mathrm{~d}$ before operation, the severe patients undergo diet limitation and catharsis, which induce dysbacteriosis and intestinal mucosal atrophy, and decrease the gut barrier function [7]. In addition, surgical trauma, stress response, and antibiotic use after operation damage the intestinal micro-ecology balance further, and aggravate dysbacteriosis and bacteria translocation. How to protect intestinal barrier function after traumatic stress becomes a difficult problem for nutrition therapy. Gln, an essential amino acid of human body, is a main energy source of gastrointestinal mucosal cells. And it can prevent intestinal mucosal atrophy, promote secretion of gastrointestinal hormones, and increase immunoglobulin of intestinal mucosa [8]. What's more, it also can restore the damage, maintain the structure and improve permeability of intestinal mucosa, and prevent the translocation of intestinal bacteria and toxin. In recent years, the protecting effect of probiotics for intestinal barrier function is concerned increasingly. Probiotics refer to live micro-organisms which can improve micro-ecology of human body and confer a health benefit on the host. The common intestinal probiotics include Bacillus bifidus and Lactobacillus [9]. Studies show that probiotics can regulate intestinal flora, slow the growth of pathogenic bacteria, reduce the translocation of intestinal bacteria and endotoxin after operation, and decrease the occurrence of infection [9-11]. So it can be used for regulating immune response, food allergy, and inflammatory bowel disease.

In a conclusion, combining probiotics with Gln can increase the amount of Lactobacillus, slow the growth of gram-negative bacterium, improve the structure of intestinal flora, and restore the damage of colonic ecological barrier. Besides, it also can lower the permeability of intestinal mucosa and the level of intestinal endotoxin, restore the damaged colonic mechanical barrier, thus reduce the translocation of intestinal bacteria. But, the amount of gram-negative bacterium doesn't decrease greatly. On this basis, whether other nutrients, such as prebiotics [12,13], are added to decrease gram-negative bacterium, improve diversity of intestinal flora, and enhance immunity, is deserved to study further.

\section{References}

1. Yin SM, Zheng BS, Zhang W. Clinical characteristics of digestive system dysfunction in the elderly with multiple organ dysfunction syndrome. Chin J Mult Organ Dis Eld 2011; 10: 36-39.

2. Tang MG, Qiu F. Protection of intestinal mucosal barrier in critical patients. J North China Coal Med Coll 2007; 9: 348-351.

3. Wang M, Li J. The effect of compound glutamine and probiotics on intestinal barrier in decompensated hepatitic cirrhosis patients. Chin J Clin Gastroenterol 2013; 39-41. 
4. Diao YP, Chen H, Li F. Importance of early intestinal barrier protection in severe acute pancreatitis. Chin J Hepatobiliary Surg 2011; 17: 462-465.

5. Zhang CL, Chen QP, Zhang XY. Evaluation and clinical application of enteral immuno-nutrition. Chin J Clinicians 2011; 201: 5 .

6. Yoshida S, Kaibara A, Ishibashi N, Shirouzu K. Glutamine supplementation in cancer patients. Nutrition 2001; 17: 766-768.

7. Shao F, Zhang YB, Yang DG. Effect of Microecpharm aceutics and glutamine enhanced microbial immune entreral nutrition on postoperative stress status and immune function in colon cancer patients. Parenter Enteral Nutr 2011; 18: 215-217.

8. Xu JJ, Lin C, Wang TT. Effect of glutamine particle on immune function of patients with severe burn injury. Chongqing Med J 2007; 36: 472-473.

9. Hao J, Cai DL. Advances in clinical application of gastrointestinal probiotic bacteria. Amino Acid Biotic Resour 2011; 33: 67-70.

10. Shen Z, Ma L. Effects of probiotics and glutamine on intestinal microorganisms of patients under diarrhea irritable bowel syndrome. Pract Pharm Clin Rem 2014; 17.
11. Wang B, Song HY, Yang JR. Progress on intervening dysfunction of intestinal barrier. World Chin J Digestol 2011; 19: 2251-2256.

12. de Moreno de LeBlanc A, Matar C, Perdigon G. The application of probiotics in cancer. $\mathrm{Br} \mathrm{J}$ Nutr 2007; 98: 105-110.

13. Sun ZH, Shi R, Zhang QL. Application of prebiotics in improving intestinal barrier function $[\mathrm{C}] / /$. Compilation of papers in 4th nutrition conference of national integrated Chinese and Western medicine 2013.

\section{*Correspondence to}

Baohua Wang

The Affiliated Hospital of North China University of Science and Technology

PR China 